\title{
A BAND MODEL FORMULATION FOR VERY NONUNIFORM PATHS*
}

\author{
G. H. Lindquist and F. S. Simmons $\dagger$ \\ Willow Run Laboratories, Institute of Science and Technology, The University of Michigan, \\ Ann Arbor, Michigan 48107, U.S.A.
}

(Received 21 July 1971)

\begin{abstract}
Anomalous behavior has been observed when molecular band-models incorporating the CurtisGodson or similar approximations are applied to problems characterized by a large variation in temperature along the optical path. The nature of this misbehavior has been examined and a procedure has been developed for its suppression by the introduction of a less restrictive assumption in the derivation of the band model from the basic equation of transfer.
\end{abstract}

\section{NOMENCLATURE}

$A, B, C, D, E$ constants used in analytic form for $\eta$

$d \quad$ average line spacing

$F \quad$ function providing interpolation between nearly weak and nearly strong approximations

$f(x) \quad$ Ladenburg-Reiche function

$g\left(v^{\prime}-v\right) \quad$ spectrometer slit function

$k$ absorption coefficient

$k$

$L_{v}$

averaged absorption coefficient for band model

spectral radiance, $W /\left(\mathrm{cm}^{2}-\mathrm{sr}-\mathrm{cm}^{-1}\right)$

black body spectral radiance, $W /\left(\mathrm{cm}^{2}-\mathrm{sr}-\mathrm{cm}^{-1}\right)$

black body spectral radiance of ith path element

spectral radiance computed using a band model

number of path elements in sum

line strength

equivalent line strength

equivalent width, $\mathrm{cm}^{-1}$

optical depth coordinate, $\mathrm{gm} \mathrm{cm}^{-2}$

total optical depth of path of interest, $\mathrm{gm} \mathrm{cm}^{-2}$

optical depth coordinate of $i$ th path element, $\mathrm{gm} \mathrm{cm}^{-2}$

dimensionless optical depth for Curtis-Godson approximation

dimensionless optical depth for nearly weak-nearly strong approximation

line overlap parameter, $2 \pi y / d$

line overlap parameter averaged over the path extending from the observer to optical depth $X$

line overlap parameter averaged over the whole optical depth of interest

line width, $\mathrm{cm}^{-1}$

line width averaged over the path extending from the observer to optical depth $X$

line width averaged over the whole optical depth of interest

interpolation parameter between nearly weak-nearly strong approximation

* This work was supported in part by NASA under Contract NAS3-13037.

+ Present address: The Aerospace Corp., El Segundo, California, U.S.A. 


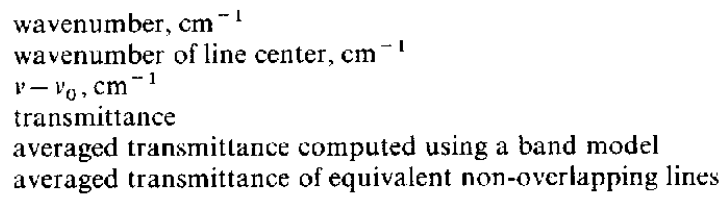

\section{INTRODUCTION}

A RECENT note ${ }^{(1)}$ was concerned with the application of a molecular band model for inhomogeneous radiating gases ${ }^{(2,3)}$ to cases in which large variations in temperature are encountered along the optical path. The problem considered was calculation of thermal radiation from a small combustion-product source viewed through a long atmospheric path. The band model used was originally formulated in terms of a nearly-weak or a nearlystrong (NW-NS) line approximation. A simple procedure for interpolation between the two approximations provided for intermediate optical depths. Applications of this band model in its original form yielded anomalous results; at some frequencies, the calculated values of apparent radiance with atmospheric absorption exceeded those for the hot source alone. Reasons for these inconsistencies were determined and corrections made. In the aforementioned note we presented both results of the use of the modified band model and the need for a more complex interpolation procedure. The purpose of this communication is to present the details of the revised formulation of the model, which represents a closer approximation to the exact equation of transfer than the original model, or comparable ones which have been based on the Curtis-Godson approximation.

The rationale in the development of the new model can be illustrated by considering the growth of a single, collision-broadened spectral line along a nonisothermal path. Once the formulation for such a single line is developed, it is carred over essentially unchanged to the case of a random band of overlapping lines.

The solution of the equation of transfer which describes the radiance of a single, isolated, spectral line from a general nonisothermal source under the conditions of local thermodynamic equilibrium, negligible scattering, and no significant source of radiation behind the gas, is

$$
L_{v}(v)=\int_{0}^{x_{L}} L_{v}^{*}(v, X) k\left(v-v_{0}, X\right) \exp \left[-\int_{0}^{x} k\left(v-v_{0}, X^{\prime}\right) \mathrm{d} X^{\prime}\right] \mathrm{d} X .
$$

Here $X$ is the optical depth (mass per unit cross sectional area) coordinate along the line of sight through the gas. $L_{v}^{*}(v, X)$, the blackbody spectral radiance, is a function of frequency, or equivalently wave-number, $v$, and temperature, $T=T(X) ; T$ in turn, is a function of the position along the line of sight through the gas. The spectral absorption coefficient of the single line being considered, $k\left(v-v_{0}, X\right)$, is a function of the spectral distance from the line center, $v-v_{0}$, and the optical depth coordinate along the path, $X . L_{v}(v)$ is the spectral radiance of the gaseous source at wave-number $v$ resulting from the single spectral line. $X_{L}$ is the total optical depth of the gaseous source. The total radiance from the line is obtained by integration over all frequencies:

$$
L=\int_{-\infty}^{\infty} \int_{0}^{X_{L}} L_{v}^{*}(v, X) k\left(v-v_{0}, X\right) \exp \left[-\int_{0}^{x} k\left(v-v_{0}, X^{\prime}\right) \mathrm{d} X^{\prime}\right] \mathrm{d} X \mathrm{~d}\left(v-v_{0}\right) .
$$


If we introduce the transmittance, defined as

$$
\tau\left(v-v_{0}, X\right) \equiv \exp \left[-\int_{0}^{X} k\left(v-v_{0}, X^{\prime}\right) \mathrm{d} X^{\prime}\right]
$$

and interchange the order of integration, assuming that $L_{v}^{*}(v, X)$ does not vary significantly over the frequency region involved, the equation for $L$ may be written as

$$
L=-\int_{0}^{X_{L}} L_{v}^{*}\left(v_{0}, X\right) \frac{\mathrm{d}}{\mathrm{d} X}\left[\int_{-\infty}^{\infty} \tau\left(v-v_{0}, X\right) \mathrm{d}\left(v-v_{0}\right)\right] \mathrm{d} X .
$$

The equivalent width, $W$, is defined as

$$
W(X)=1-\int_{-\infty}^{\infty} \tau\left(v-v_{0}, X\right) \mathrm{d}\left(v-v_{0}\right)
$$

so that the expression for $L$ becomes

$$
L=\int_{0}^{x_{L}} L_{v}^{*}\left(v_{0}, X\right) \frac{\mathrm{d} W}{\mathrm{~d} X} \mathrm{~d} X
$$

Since $W$ is only a function of $X$, the preceding equation may be written as

$$
L=\int_{0}^{W\left(X_{L}\right)} L_{v}^{*}\left(v_{0}, X\right) \mathrm{d} W(X)
$$

If we divide the path into $N$ approximately isothermal elements, the expression for $L$ becomes

$$
L \cong \sum_{i=1}^{N} L_{v, i}^{*}\left[W\left(X_{i}\right)-W\left(X_{i-1}\right)\right]
$$

where $X_{i}$ is the optical path from the observer to the far boundary of the $i$ th isothermal element and $W\left(X_{i}\right)$ is the equivalent width corresponding to that path.

Calculation of the values of $W\left(X_{i}\right)$ shows that, in general, the integrals over $X$ and $v-v_{0}$ are not separable for nonisothermal paths. To allow such separation, an approximation for the variation of the line shape along the path is usually made. The Curtis-Godson approximation is most commonly used for this purpose. ${ }^{(4-6)}$ Another, is the nearly-weak nearly-strong line approximation. ${ }^{(1,3)}$ The purpose of both approximations is the separation of the integrals over $X$ and $v-v_{0}$ and the replacement of the nonisothermal path with an equivalent isothermal path. Both yield satisfactory values of $W(X)$ for many cases, especially when the temperature and concentration variations are not too large.

In the evaluation of equation (6), the accuracy of the integration depends not so much on the accuracy of the values of $W(X)$, but on the accuracy of differences between values of $W(X)$, i.e. on the derivative of $W$ with respect to $X$. However, an expression which is a reasonable approximation for $W$ will not always yield a reasonable approximation to 
$\mathrm{d} W / \mathrm{d} X$. To illustrate this, we will compute the derivative of $W$ with respect to $X$, using both the Curtis-Godson approximation and the NW-NS approximation.

First it is necessary to obtain an expression for the equivalent width of a collisionbroadened line in an isothermal path. The absorption coefficient in this case is

$$
k\left(v-v_{0}, X^{\prime}\right)=\frac{S\left(X^{\prime}\right)}{\pi} \begin{gathered}
\gamma\left(X^{\prime}\right) \\
\gamma^{2}\left(X^{\prime}\right)+\left(v-v_{0}\right)^{2}
\end{gathered}
$$

where $S$ is the line strength and $\gamma$ its half-width. Upon substitution into equation (3) specialized to an isothermal path, and with the use of equation (4) the following expression for $W$ is obtained after some reduction :?

$$
W(X)=2 \pi \gamma \cdot f(x)
$$

where $f$ is the Ladenburg-Reiche function,

$$
f(x) \equiv x \mathrm{e}^{-x}\left[J_{0}(i \cdot x)-i J_{1}(i \cdot x)\right]
$$

and $x=S X / 2 \pi \gamma$ is the dimensionless optical depth. $J_{0}$ and $J_{1}$ are Bessel functions of the first kind of order 0 and 1 respectively. Tables of $f(x)$ are found in the literature ${ }^{(8,9)}$

We have used the form of the Curtis-Godson approximation described by Goony. ${ }^{(4)}$ It is a method in which the equivalent width of an absorption line as viewed through a nonisothermal path can be approximated by the equivalent width through some corresponding isothermal path. The approximation involves the definition of an equivalent average half-width and line strength for the path extending from the observer to some point, $X$, in the path, viz.

$$
S_{e}(X)=\frac{\left[\int_{0}^{X} S\left(X^{\prime}\right) \mathrm{d} X^{\prime}\right]}{X}
$$

and

$$
\gamma_{e}(X)=\frac{\int_{0}^{X} \gamma\left(X^{\prime}\right) S(X) \mathrm{d} X^{\prime}}{\int_{0}^{X} S\left(X^{\prime}\right) \mathrm{d} X^{\prime}}
$$

The denominator in the nonisothermal absorption coefficient, given by equation (7), is taken to be independent of $X^{\prime}$, of the form $\gamma_{e}^{2}(X)+\left(v-v_{0}\right)^{2}$, so that

$$
\int_{0}^{X} k\left(v-v_{0}, X^{\prime}\right) \mathrm{d} X^{\prime}=\frac{\gamma_{e}(X) S_{e}(X) X}{\pi\left[\gamma_{e}^{2}(X)+\left(v-v_{0}\right)^{2}\right]} .
$$

It now follows that

$$
W(X)=2 \pi \gamma_{e}(X) f(x),
$$

where $x$, the dimensionless optical depth, is defined as

$$
x(X) \equiv \frac{\int_{0}^{X} S\left(x^{\prime}\right) \mathrm{d} X^{\prime}}{2 \pi \gamma_{e}(X)} .
$$


Equation (10) can now be used to investigate the behavior of $\mathrm{d} W / \mathrm{d} X$ in a nonisothermal gas. If we differentiate equation (10) and make use of equation (8) we get

$$
\frac{\mathrm{d} W}{\mathrm{~d} X}=S(X)\left\{\frac{\mathrm{d} f(x)}{\mathrm{d} x}\left[2-\frac{\gamma(X)}{\gamma_{e}(X)}\right]+\frac{f(x)}{x}\left[\frac{\gamma(X)}{\gamma_{e}(X)}-1\right]\right\} .
$$

The variation with optical depth $X$ may be mapped into a variation with dimensionless optical depth, $x$, and equation (12) rewritten as

$$
\frac{1}{S(X)} \frac{\mathrm{d} W}{\mathrm{~d} X}=\left\{\frac{\mathrm{d} f(x)}{\mathrm{d} x}\left[2-\frac{\gamma}{\gamma_{e}}(x)\right]+\frac{f(x)}{x}\left[\frac{\gamma}{\gamma_{e}}(x)-1\right]\right\}
$$

We can now place physical limitations on $[1 / S / X)](\mathrm{d} W / \mathrm{d} X)$. At $X=0$, the quantity $[1 / S(X)](\mathrm{d} W / \mathrm{d} X)$ must be unity corresponding to the region of linear growth for small optical depths. It must also be less than one at larger optical depths as the square root region is approached; and, finally, it must remain non-negative for all $x$, so that the equivalent width continues to grow as $x$ increases. The variation of $[1 / S(X)](\mathrm{d} W / \mathrm{d} X)$ in equation (13) is shown in Fig. 1 as a function of $x$ with $\gamma / \gamma_{e}$ as a parameter. $\gamma / \gamma_{e}$ is the ratio of the half-width at $x$ to its average value taken over all the path before it. For $\gamma / \gamma_{e} \leq 2$, the function is well behaved, but not for $\gamma / \gamma_{e}>2$. In the latter case the function becomes larger than one for intermediate values of $x$, indicating a rate of equivalent-width increase greater than that in the linear region. These errors, mentioned by GoODY ${ }^{(4)}$ are treated in detail by Drayson. ${ }^{(5)}$ However, the analyses dealt only with the absorption (or equivalent width). On the other hand WaLsHaw and RoDGers, ${ }^{(10)}$ analyzed the effect of the CurtisGodson approximation on the derivative of transmittance with optical depth, for several band models.

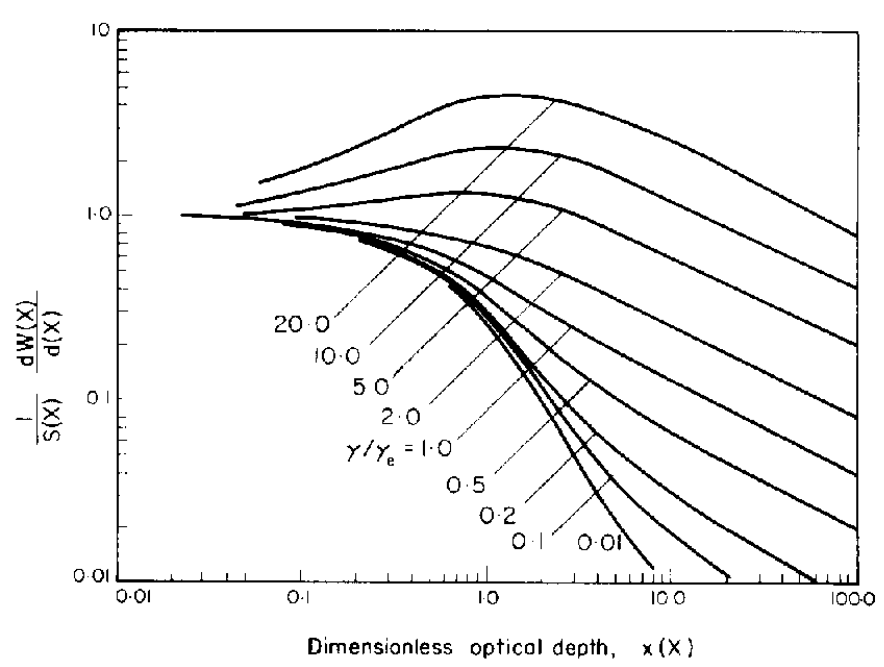

Fig. 1. Equivalent width derivative in the Curtis-Godson approximation. 


\section{NEARLY-WEAK-NEARLY-STRONG LINE APPROXIMATION}

This approximation, like the Curtis-Godson approximation, defines an average for the line half-width. However, this average is fixed for the path in question and is defined as the average over the total path, $X_{L}$ :

$$
\gamma_{e L}=\frac{\int_{0}^{X_{L}} \gamma\left(X^{\prime}\right) S\left(X^{\prime}\right) \mathrm{d} X^{\prime}}{\int_{0}^{X_{L}} S\left(X^{\prime}\right) \mathrm{d} X^{\prime}} .
$$

Then the dimensionless optical depth is defined as

$$
\bar{x}(X)=\frac{1}{2 \pi \gamma_{e L}} \int_{0}^{X} S\left(X^{\prime}\right) F\left(X^{\prime}\right) \mathrm{d} X^{\prime} .
$$

$F\left(X^{\prime}\right)$ is a function which must be unity in the nearly weak approximation and $\gamma / \gamma_{e L}$ in the nearly strong approximation. ${ }^{(11)} \mathrm{A}$ form must be chosen for the variation of $F\left(X^{\prime}\right)$ so that it produces a reasonable transition between the two approximations for intermediate optical depths. The equivalent width is given again as

$$
W(X)=2 \pi \gamma_{e L} f(\bar{x}) .
$$

If we take the derivative of equation (16) for the nearly weak approximation we obtain

$$
\frac{\mathrm{d} W}{\mathrm{~d} X}=S(X) \frac{\mathrm{d} f(\bar{x})}{\mathrm{d} \bar{x}},
$$

and for the nearly strong approximation, we obtain

$$
\frac{\mathrm{d} W}{\mathrm{~d} X}=S(X) \frac{\gamma(X)}{\gamma_{e L}} \frac{\mathrm{d} f(\bar{x})}{\mathrm{d} \bar{x}} .
$$

Values for $[1 / S(X)](\mathrm{d} W / \mathrm{d} X)$, according to equations (17) and (18) are shown in Fig. 2. It can be seen that the nearly weak approximation never becomes physically unrealistic, i.e.

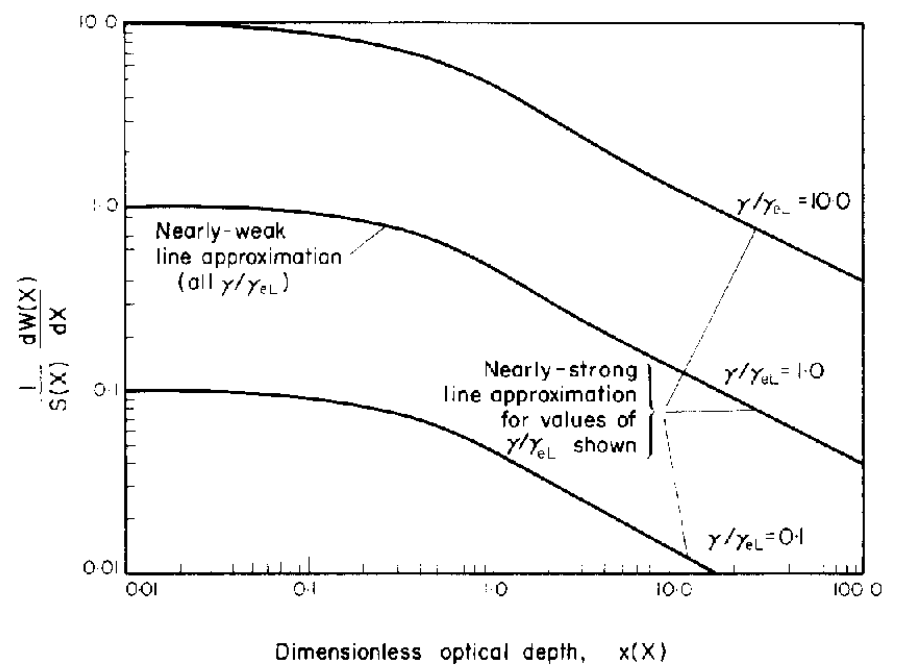

Fir. 2. Equivalent width derivative in the nearly-weak and nearly-strong line approximations. 
$[1 / S(X)](\mathrm{d} W / \mathrm{d} X) \leq 1$; however, for the nearly strong approximation, the quantity $[1 / S(X)](\mathrm{d} W / \mathrm{d} X)$ becomes greater than one at small values of $\bar{x}$ for $\gamma(X) / \gamma_{e L}>1$. This is accounted for by the proper choice of $F\left(X^{\prime}\right)$. However, we are left with no information upon which to base $F\left(X^{\prime}\right)$ at this stage.

It appears that neither the nearly weak-nearly strong approximation (unless $F(X)$ is accurately specified) nor the Curtis-Godson approximation give sufficiently accurate values for $\mathrm{d} W / \mathrm{d} X$ over the full range of optical depths and for all possible values of $\gamma / \gamma_{e}$. On the other hand, it should be noted that the anomalies and errors in the curves for $(1 / S)(\mathrm{d} W / \mathrm{d} X)$ as given by the Curtis-Godson approximation are not serious as long as $\gamma / \gamma_{e}$ never exceeds a value of about two. This condition exists ${ }^{(4,5,10)}$ for most atmospheric paths but not for highly nonhomogeneous paths. For example, hot products of combustion viewed through long atmospheric paths yield values of $\gamma / \gamma_{e}$ as large as 20 .

To obtain a more acceptable expression for $\mathrm{d} W / \mathrm{d} X$, consider the growth of an isolated spectral line. The equivalent width is given exactly by

$$
W(X)=1-\int_{-\infty}^{\infty} \exp \left[-\int_{0}^{x} k\left(\Delta v, X^{\prime}\right) \mathrm{d} X^{\prime}\right] \mathrm{d}(\Delta v)
$$

and its derivative, in terms of a Lorentz profile, by

$$
\frac{\mathrm{d} W}{\mathrm{~d} X}=\frac{1}{\pi} \int_{-\infty}^{\infty} \frac{S(X) \gamma(X)}{\gamma^{2}(X)+(\Delta v)^{2}} \exp \left[-\frac{1}{\pi} \int_{0}^{X} \frac{S\left(X^{\prime}\right) \gamma\left(X^{\prime}\right)}{\gamma^{2}\left(X^{\prime}\right)+(\Delta v)^{2}} \mathrm{~d} X^{\prime}\right] \mathrm{d}(\Delta v)
$$

where $\Delta v=v-v_{0}$. In the original treatment of the Curtis-Godson and the nearly weaknearly strong approximation, the separation of frequency-dependent and path-dependent variables was accomplished by substitution of an effective value, $\gamma_{e}$, in both parts of the integrand. The only difference in our treatment from previous treatments is that we will use such an approximation only in the exponential term. Thus, in terms of $\gamma_{e}=\gamma_{e}(X)$ defined by equation ( 8 ) and $x$ defined by equation (11), equation (20) becomes

$$
\frac{\mathrm{d} W}{\mathrm{~d} X}=\frac{1}{\pi} \int_{-\infty}^{\infty} \frac{S(X) \gamma(X)}{\gamma^{2}(X)+(\Delta v)^{2}} \exp \left[-2 x \gamma_{e}(X) \frac{\gamma_{e}(X)}{\gamma_{e}^{2}(X)+(\Delta v)^{2}}\right] \mathrm{d}(\Delta v) .
$$

This is equivalent to making a Curtis-Godson substitution in the expression for the derivative of $W$, rather than in the expression for $W$ itself. The process of taking a derivative after an approximation has been made is reversed, a treatment which should be inherently better than the techniques described earlier for the calculation of radiances. This new approximation can be compared to the previous two if $\mathrm{d} W / \mathrm{d} X$ is considered as the limiting value of the contribution to $W$ from a small element of path, $\Delta X$, viewed through the path ahead of it. In the previous two approximations, the line shape of the element $\Delta X$ and the path in front of it are both given by the line shape for the equivalent, homogeneous path. In the current approximation we assumed that the element $\Delta X$ has its true line shape, while the path in front of it has an equivalent homogeneous shape. Equation (21), checked against the exact relation, equation (20), for a two-layer, nonisothermal path, has been found to be very accurate. Further investigation of its accuracy is to be the subject of future work. It 
would be possible to fully develop a procedure for treating isolated Lorentz lines. However, our interest is ultimately in a random band model which treats the average of many lines.

\section{BAND-MODEL CONSIDERATIONS}

The calculation of an average spectral radiance at the boundary of a radiating body of gas, in a frequency interval encompassing a number of rotational lines, is represented by the relation

$$
\bar{L}_{v}=\int_{0}^{\infty} g\left(v^{\prime}-v\right) L_{v}\left(v^{\prime}\right) \mathrm{d} v^{\prime},
$$

where $g\left(v, v^{\prime}\right)$ is an averaging function, e.g. the slit function of a spectrometer of moderate resolution, such as those which produce original absorption spectra from which band-model parameters are extracted. The exact spectral radiance, $L_{v}$, is given by the equation of transfer, equations (1) and (3). Thus, equation (22) can be written as

$$
\bar{L}_{v}=\int_{0}^{\infty} g\left(v^{\prime}-v\right) \int_{0}^{\tau\left(X_{I}\right)} L_{v}^{*}\left(v, \tau^{\prime}\right) \mathrm{d} \tau^{\prime} \mathrm{d} v^{\prime},
$$

where $\tau$ is the perfectly resolved transmittance, and the primes denote variables of integration.

The order of integration in equation (23) can be inverted, and when frequency intervals are small enough that the Planck function is cssentially constant, the result is the band-model expression :

$$
\bar{L}_{v}(v)=\int_{1}^{\bar{\tau}\left(X_{L}\right)} L_{v}^{*}\left(v, \bar{\tau}^{\prime}\right) \mathrm{d} \bar{\tau}^{\prime}
$$

where

$$
\bar{\tau}(v) \equiv \int_{0}^{x_{1}} g\left(v^{\prime}-v\right) \tau\left(v^{\prime}\right) \mathrm{d} v^{\prime} .
$$

Thus, $\bar{\tau}$ is seen to be the spectral transmittance measured by a conventional instrument or calculated by a band-model.

Equation (25) can be evaluated to obtain the average spectral radiance, $\bar{L}_{v}$, by first computing $\bar{\tau}$ at a number of points along the path. The blackbody radiance is then represented as a function of $\bar{\tau}$, with, for instance, the use of a simple summation. The various band models in current use, including those based on the Curtis-Godson approximation and the present model in its original form, do yield values for the transmittance with an accuracy sufficient for most engineering applications. However, as for the case of isolated lines, equation (25) involves a differentiation of the expression for $\bar{\tau}$ with respect to the optical path. As in the case of an isolated line, an expression which yields reasonable values for $\bar{\tau}$ will not necessarily yield reasonable values for $\mathrm{d} \bar{\tau}$ and, hence, for $\bar{L}_{v}$. It was 
necessary, therefore, to develop an expression for $\bar{\tau}$ which has a derivative which will yield realistic values of the difference in transmittance with a specified incremental increase in physical path.

The transmittance for a band of spectral lines with some overlapping may be expressed $\mathrm{as}^{(4)}$

$$
\bar{\tau}=\exp \left(\bar{\tau}_{n}-1\right)
$$

where $\bar{\tau}_{n}$ is the transmittance for an equivalent band of well-isolated nonoverlapping lines. Differentiation of equation (26) with respect to the optical depth, $X$, yields

$$
\frac{\mathrm{d} \bar{\tau}}{\mathrm{d} X}=\bar{\tau} \frac{\mathrm{d} \bar{\tau}_{n}}{\mathrm{~d} X}
$$

which indicates that, with a reasonably accurate value for $\bar{\tau}$, the accuracy of $\mathrm{d} \bar{\tau} / \mathrm{d} X$ depends directly on the accuracy of $\mathrm{d} \bar{\tau}_{n} / \mathrm{d} X$.

In our current work, we have used the NW-NS approximation exclusively and at this point our consideration is limited to this model to find specification of the function $F\left(X^{\prime}\right)$ which yields accurate values of a transmittance derivative. For this approximation ${ }^{(11)}$

$$
\bar{\tau}_{n}=\beta_{e L} f(\bar{x}),
$$

where the argument of the Ladenburg-Reiche function is, in this case

$$
\bar{x}(X)=\frac{1}{\beta_{e L}} \int_{0}^{X} \bar{k}\left(X^{\prime}\right)\left[\frac{\beta\left(X^{\prime}\right)}{\beta_{e L}}\right]^{\eta\left(X^{\prime}\right)} \mathrm{d} X^{\prime} .
$$

Here $\bar{k}=\bar{k}(v, T)$ is the first band-model parameter, the average absorption coefficient, identified as the average line strength to spacing ratio, and $\beta=\beta(v, T)$ is the second bandmodel parameter, the line-overlap factor, identified as $2 \pi$ times the average line width to spacing ratio. The effective value, $\beta_{e L}$, is defined analogously to $\gamma_{e L}$ for the case of a single line:

$$
\beta_{e L}=\frac{\int_{0}^{X_{I_{I}}} \bar{k}\left(X^{\prime}\right) \beta\left(X^{\prime}\right) \mathrm{d} X^{\prime}}{\int_{0}^{X_{L}} k\left(X^{\prime}\right) \mathrm{d} X}
$$

$F\left(X^{\prime}\right)$ has here been replaced by $\left[\beta\left(X^{\prime}\right) / \beta_{e L}\right]^{\eta\left(X^{\prime}\right)}$, a suitable functional form for $F\left(X^{\prime}\right)$. Here $\eta$ is an interpolation parameter between the nearly weak and nearly strong approximations, varying from 0 for the nearly weak to 1 for the nearly strong.

In an earlier study, ${ }^{(1)}$ an investigation of the form for $\eta$ is described. As a result of that study, several reasonably useful empirical forms for $\eta$ were determined. However, the information used to make these determinations was not sensitive enough to values of $\eta$ to provide accurate information about its form. One of these empirical forms is: $\eta=0$ for $\bar{x}<1, \eta=(\bar{x}-1) / 9$ for $1 \leq x \leq 10, \eta=1$ for $\bar{x}>10$. This appears to make $\eta$ a function of $\bar{x}$ rather than $X^{\prime}$, so that the determination of $\bar{x}$ appears as an iterative procedure. However, in practice, the integral for $\bar{x}$ is evaluated by a summation and a value for $\eta$ for a particular term is determined from the value for $\bar{x}$ calculated from the sum of the preceding terms. Thus, $\eta$ is uniquely defined for each $X^{\prime}$. 
Differentiation of equation (28) and utilization of equation (30) yields

$$
\frac{\mathrm{d} \bar{\tau}_{n}}{\mathrm{~d} X}=\frac{\mathrm{d} f}{\mathrm{~d} \bar{x}}\left[\frac{\beta(X)}{\beta_{e L}}\right]^{n(X)} \bar{k}(x),
$$

which shows that the derivative of $\mathrm{d} \bar{\tau}_{n} / \mathrm{d} X$ varies with the absorption coefficient, (as would be expected), the derivative of the function $f(\bar{x})$, and the local values of $\beta(X)$ and $\eta(X)$. The physical significance of equation (31) can be seen in Fig. 3, a normalized plot of $-(1 / \bar{k}) \mathrm{d} \tau_{n} /$ $\mathrm{d} X$ vs. $x$, with $\beta / \beta_{e L}$ as a parameter. In the limit, as $x \rightarrow 0$, the band model reduces to a linear growth law, so that $-(1 / k) \mathrm{d} \bar{\tau}_{n} / \mathrm{d} X$ approaches unity. This function must be less than unity for all values of $X$ greater than zero, as the square root growth law becomes operative. However, Fig. 3 shows that the empirical specifications for $\eta$ do not conform to this requirement for certain values of $\beta / \beta_{e L}$ and $\bar{x}$, an inconsistency analogous to that shown earlier for isolated line growth. $\mathrm{d} \bar{\tau}_{n} / \mathrm{d} X$ is correctly given by ${ }^{(4)}$

$$
\frac{\mathrm{d} \bar{\tau}_{n}}{\mathrm{~d} X}=-\frac{1}{d} \cdot \frac{\mathrm{d} \bar{W}}{\mathrm{~d} X}
$$

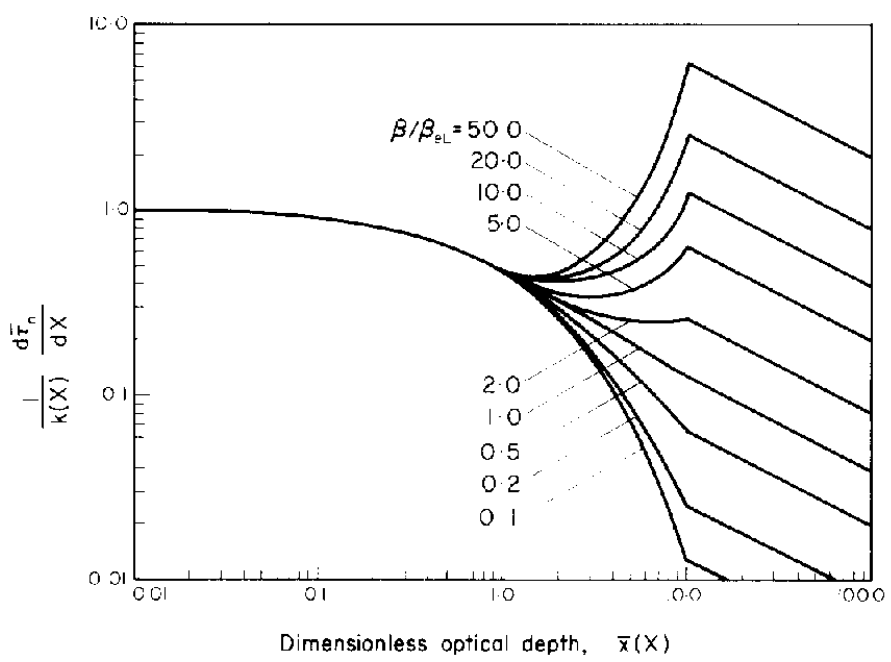

FIG. 3. Transmittance derivative in the original band-model.

where $\bar{W}$ is an average width and $d$ is an average line spacing in the spectral interval under consideration. For lines of equal strength and width, after some rearrangement, equations (32) and (21) yield

$$
\frac{\mathrm{d} \bar{\tau}_{n}}{\mathrm{~d} X}=-\frac{1}{\pi} \int_{-\infty}^{\infty} \frac{\bar{k}(X) \beta(X)}{\beta^{2}(X)+(2 \pi \Delta v / d)^{2}} \exp \left[-2 x \beta_{e}(X) \frac{\beta_{e}(X)}{\beta_{e}^{2}(X)+(2 \pi \Delta v / d)^{2}}\right] \mathrm{d}\left(\frac{2 \pi \Delta v}{d}\right),
$$

where $x$ denotes the dimensionless optical depth as defined in equation (11) and used in equation (21). Rewritten in terms of the two bandmodel parameters,

$$
x=\frac{\int_{0}^{X} \bar{k}\left(X^{\prime}\right) \mathrm{d} X^{\prime}}{\beta_{e}(X)}
$$


where $\beta_{e}(X)$ is the average value of the second band-madel parameter over the path from the observer to the point $X$, similar to the definition of $\gamma_{e}(X)$ in equation (8); thus,

$$
\beta_{e}(X)=\frac{\int_{0}^{X} k\left(X^{\prime}\right) \beta\left(X^{\prime}\right) \mathrm{d} X^{\prime}}{\int_{0}^{X} k\left(X^{\prime}\right) \mathrm{d} X^{\prime}} .
$$

The distinction between $\beta_{e L}$ (a constant determined over the whole path), defined in equation (3) and $\beta_{e}(X)$ (a function of $X$, the optical depth coordinate) should be noted.

The change of variables $\tan \theta / 2=2 \pi\left(\Delta v / \beta_{e} d\right)$ and a trigonometric substitution yields

$$
-\frac{1}{\bar{k}(X)} \cdot \frac{\mathrm{d} \bar{\tau}_{n}}{\mathrm{~d} X}=\frac{2}{\pi} \frac{\beta}{\beta_{e}} \int_{0}^{\pi} \frac{\exp [-x(1+\cos \theta)]}{\left[\left(\frac{\beta}{\beta_{e}}\right)^{2}+1\right]+\left[\left(\frac{\beta}{\beta_{e}}\right)^{2}-1\right] \cos \theta} \mathrm{d} \theta .
$$

Equation (36) has been integrated numerically; the results are plotted in Fig. 4 as $-(1 / \bar{k}) \mathrm{d} \bar{\tau}_{n} /$ $\mathrm{d} X$ versus $x ; \beta(X) / \beta_{e}(X)$ is the parameter. The resulting curves exhibit none of the anomalies seen in Fig. 3 ; the curve for $\beta / \beta_{e}=1$ is identical in both figures.

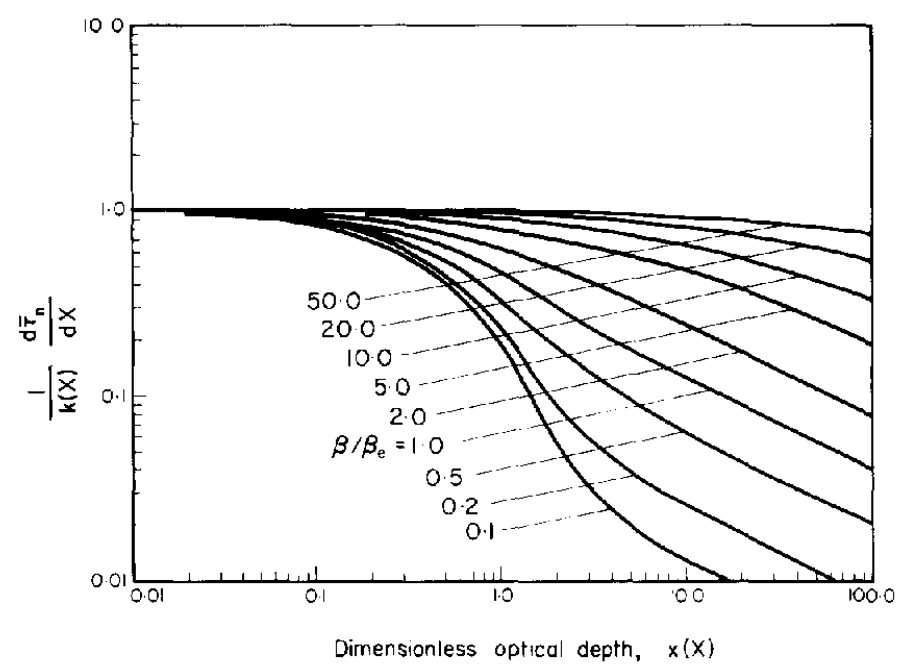

FIG. 4. Transmittance derivative in the modified band-model.

The interpolation parameter, $\eta$, can now be evaluated by equating equations (36) and (31). These two equations involve different sets of variables, i.e. equation (36) is given in terms of $x$ and $\beta_{\mathrm{e}}(X)$, and equation (31) is given in terms of $\bar{x}$ and $\beta_{e L}$. The relationship between $\bar{x}$ and $x$ is complex and depends on $\eta, \beta_{e L}(X)$ and $\beta_{e L}$ in an integral form. Furthermore, although $x$ is a unique function of the optical path extending from 0 to $X, \bar{x}$ is not a unique function in the same sense, since it depends on the path between $X$ and $X_{L}$ through $\beta_{e L}$ as well. Such dependence is not physically realistic but appears as an integral part of the NW-NS approximation. In determining a form for $\eta$, this dependence must be accounted for so that the computed value of $(1 / \bar{k})\left(\mathrm{d} \tau_{n} / \mathrm{d} X\right)$ is unique despite the nonunique character 
of $\bar{x}$, although this is very difficult to ensure, except for certain types of paths. In particular, if $\beta$ differs significantly from its average value, $\beta_{e L}$, in only a very small portion of the optical path, then the contribution from that portion of the path to the integral defining $\bar{x}$ is negligible compared to the contributions from the remainder of the path. In this case, the following approximate relation holds:

$$
\int_{0}^{X} k\left(X^{\prime}\right)\left[\frac{\beta\left(X^{\prime}\right)}{\beta_{e I .}}\right]^{n\left(X^{\prime}\right)} \mathrm{d} X^{\prime} \simeq \int_{0}^{X} \bar{k}\left(X^{\prime}\right) \mathrm{d} X^{\prime} .
$$

The above restriction also implies that $\beta_{e}(X)=$ constant $=\beta_{e L}$, and that $x \simeq \bar{x}$. Thus, if $\beta_{e}(X)$ is not a strong function of $X$ and if large variations in $\beta(X)$ are allowed in very small fractions of the optical path only, values for $\eta$ can be found in terms of $\bar{x}$ and $\beta / \beta_{e L}$ (except for $\beta / \beta_{e L}=1$ where $\eta$ is indeterminate). The resultant values of $\eta$ are shown in Fig. 5 . Use of these values in equation (31) will insure that the derivative $\mathrm{d} \tau / \mathrm{d} X$ will equal that given by equation (36). For convenience in application, we made simple analytical approximations to the curves of Fig. 5 by fitting them with functions as follows:

$$
\begin{aligned}
\eta\left(\bar{x}, \beta / \beta_{e}\right) & =\frac{\bar{x}+A\left(\beta^{\prime} / \beta_{e}\right)}{x+B\left(\beta / \beta_{e}\right)}, \\
\eta\left(\bar{x}, \beta / \beta_{e}\right) & =\frac{\bar{x} C\left(\beta / \beta_{e}\right)}{E\left(\beta / \beta_{e}\right)+x D\left(\beta / \beta_{e}\right)} .
\end{aligned}
$$

For any particular instance, the choice between equations (37) and (38) is made on the basis of the quantity

$$
E\left(\beta / \beta_{e}\right)=1+0.185 \beta / \beta_{c} .
$$

If $\bar{x}>E$, equation (37) is used; otherwise equation (38) is used. Values for $A, B, C$ and $D$ are tabulated in Table 1 . Values of $\mathrm{d} \bar{\tau}_{n} / \mathrm{d} X$ obtained when the values in Table 1 are used in place of the values shown for $\eta$ in Fig. 5 are sufficiently accurate for most applications of the band-model.

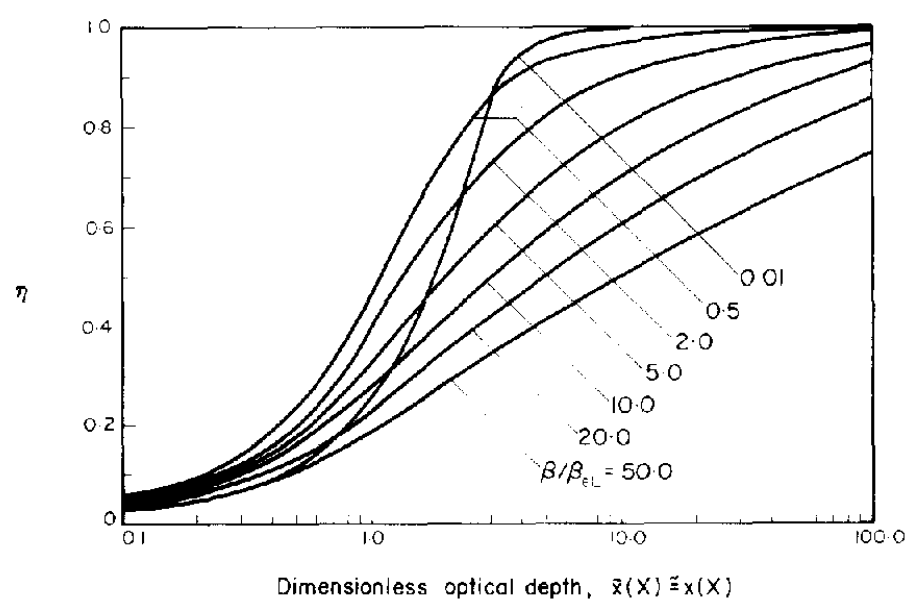

FIG. 5. Interpolation parameter as a function of optical depth and line overlap factor. 
Table 1. Coefficients to Be USED IN equations (37) AND (38)

\begin{tabular}{rcccc}
\hline$\beta / \beta_{e}$ & $A$ & $B$ & $C$ & $D$ \\
\hline 0 & -0.68 & -0.35 & 0.55 & 0.07 \\
2 & -0.37 & 0.62 & 0.71 & 0.43 \\
5 & 0.60 & 3.15 & 0.90 & 0.79 \\
10 & 3.19 & 9.25 & 1.15 & 1.30 \\
20 & 9.10 & 22.8 & 1.63 & 2.26 \\
50 & 29.1 & 67.1 & 2.78 & 4.56 \\
100 & 105.0 & 209.0 & 4.05 & 7.09 \\
\hline
\end{tabular}

The limitation on the variation of $\beta(X)$ and $\beta_{e}(X)$ mentioned earlier is not as severc as it might first appear. For instance, in cases where a relatively small hot gaseous source is to be viewed through a long, cool path, the value of $\beta$ is almost invariant over the whole atmospheric path which contains the largest part of the optical path. Only in the relatively small portion of the path comprising the hot gaseous source does $\beta$ vary substantially.

The introduction of an interpolation parameter has little physical basis and is not the only way equation (36) could be introduced into a computational procedure. An alternative method for use of the above results would be the direct application of equation (36) into a band-model computer code. The resultant values of $\mathrm{d} \bar{\tau}_{n} / \mathrm{d} X$ could then be used in equation (27), if $\bar{\tau}_{n}$ in equation (26) is evaluated in terms of $x$ and $\beta_{e}(X)$. Such a procedure would eliminate the need for a distinction between the NW and NS approximations and, therefore, eliminate an interpolation procedure. Unfortunately, when equation (36) is used, an analytic form for the solution of the integral is not easily obtained. Although a solution has been obtained in the form of an infinite series, this represents little improvement over a table of numerical values. Whether there would be a net gain in computational efficiency remains to be seen. However, a direct application of equation (36) might be expected to be somewhat more satisfactory because the restriction on the variation of $\beta_{e}(X)$ compared to $\beta_{e L}$ required in the evaluation of $\eta$ would not be needed. This alternative mode of the band-model formulation is one of the subjects of a continuing investigation.

In summary, we have shown how both the Curtis-Godson approximation and the NW-NS approximation can introduce anomalies into the radiance computed over a highly nonisothermal path for an isolated spectral line. The application of a Curtis-Godson approximation to the expression for the equivalent-width gradient along the path produced a formulation yielding greatly improved radiance values which exhibit no anomalies. In the generalization to a random band model, the expression concerning the equivalentwidth gradient has been used to obtain an interpolation between the nearly-weak and nearly-strong approximations valid for that band model.

The most important result presented herein is the form of the transmittance gradient given in equation (36). It is the basis of a computational procedure for computing the radiance from highly nonisothermal gaseous sources. This procedure yields significant improvements over previously used techniques. It has been shown to be a better approximation than those more commonly used and yields physically realistic results for all values of the path and band-model parameters. 


\section{REFERENCES}

1. F. S. Simmons, C. B. Arvold, G. H. Lindouist and F. G. Smith, Appl. Optics 9, 2792 (1970).

2. F. S. Simmons, Appl. Optics 5, $1801(1966) ; 6,1423$ (1967).

3. F. S. Simmons, C. B. Arnold and H. Y. Yamada, Measurement of Temperature Profiles in Hot Gases by Emission-Absorption Spectroscopy. NASA CR-72491 (1969).

4. R. M. GoODY, Atmospheric Radiation-1. Theoretical Basis. Oxford Calendar Press (1964).

5. S. R. Drayson, Appl. Optics 5, 385 (1966).

6. A. C. CODGLeY, JQSRT 10, 1065 (1970).

7. R. Ladenburg and F. Reiche, Ann. Phys. 42, 181 (1913).

8. W. M. Elsasser, Heat Transfer by Infrared Radiation in the Atmosphere. Harvard Meteorological Studies, Report No. 6, Harvard University (1942).

9. L. D. KAPLAN and D. F. EGGERS, J. chem. Phys. 25, 875 (1956).

10. C. D. Walshaw and C. D. Rodgers, R. Met. Soc. Quart. J. 89, 122 (1963).

11. F. S. SIMMONS, JQSRT 7, 111 (1967). 